\title{
ARTIFICIAL GRASS AND GENUINE FOOTBALL: THE EVOLUTION OF ARTIFICIAL TURF
}

\author{
KJETIL K. HAUGEN AND KNUT P. HEEN
}

\begin{abstract}
The article formulates, solves and draws inference from a game model intended to shed light on the evolution of artificial turf in professional European football (soccer). The main results indicate that the quality of teams (sports performance-wise) defines which of the teams choose to play on artificial turf and which not. Furthermore, teams with low quality are predicted to be the "artificial turf pioneers", both model-wise, as well as indicated by some empirical examples with data taken from the Norwegian, Swedish and Dutch leagues. The fact that artificial turf may play a significant role also in the evolution of uncertainty of outcome is interesting and commented on in the conclusion.
\end{abstract}

\section{INTRODUCTION AND LITERATURE}

The use of artificial turf in professional European football has experienced a tremendous growth since $\mathrm{QPR}^{1}[5]$ installed artificial turf on their home stadium, Loftus Road, in 1981. The fact that QPR removed the turf only 7 years later did not stop the evolution of artificial turf in other European countries.

Obviously, this technology became most popular in countries with climatic challenges. Today, more than half of the teams in both Norwegian and Swedish top leagues play on aritifical turf home grounds. Furthermore, according to [7], professional top level football on artificial turf is played in many other - and, in terms of climate, seemingly less problematic - European locations such as Belgium, Holland, France, Italy, Portugal, Russia and Switzerland.

Research on artificial turf has also grown considerably in the same time period. According to [9], such research can be divided into the three categories of infrastructure, user safety and play performance.

Infrastructure relates to the technology of artificial turf and its development. Surely, the quality of the grass substitute has developed considerably over the years, and today's version is often named third (or fourth) generation. It involves longer plastic fibres and certain rubber particles (granules) combined with sand mimicking the combination of grass on soil. It should not come as a surprise that considerable (often industry initiated) R\&D is involved in the continuing quality improvement of artificial turf.

$M S C$ (2010): primary 62C99, 62F03, 91A 80.

Keywords: game theory, artificial turf, natural turf.

Thanks to various Norwegian, Swedish and Dutch web-sites providing necessary information for the empirical parts of this article.

${ }^{1}$ Queens Park Rangers is a London based UK football club. 
The second category, user safety, involves mostly injury related (medical) research. In the early years of artificial turf, the surface was often rather hard, a fact that surely induced injury patterns different from those observed in playing on a natural grass surface. Much of this research is related to epidemiology studies; involving (typically) empirical analyses comparing injury patterns on and off artificial turf. A comprehensive review of this brand of research can be found in [13].

The third category, play performance, relates to how the game itself is affected by artificial turf. Football is a complex game, involving passing, ball movement, ball bounce, dribbling, etc. As such, the surface used for playing is obviously of importance. Some interesting articles representative for this branch of research can, for instance, be found in [8] or [11].

Although the three categories discussed above spawn quite a broad research area, there are certain relevant questions missing. What makes some clubs choose artificial turf and others stick to playing on natural grass? Does the quality of the club affect such decisions? Does the competitive level in the league have impact on the choice of turf? How will the distribution of artificial and natural turfs evolve? When (if ever) will all teams choose artificial turf? Will artificial turf die out? Some might be tempted to define this within a category named the economics of artificial turf, in this paper the term: the evolution of artificial turf is used. That is, this article aims to use a (relatively simple) game theory to shed light on some of these questions. Questions that definitely ought to be of interest for the football community in general, and producers of artificial turf in particular.

The next section (Section 2) introduces a game model, as well as solutions (finding Nash equilibria) and discussion of results. Section 3 investigates some simple empirical examples while Section 4 provides conclusions. Appendix A contains some mathematical derivations supporting Figure 2.

\section{BASIC MODEL}

Let us assume that two football teams $T_{1}$ and $T_{2}$ are facing a match (or in reality a sequence of at least two matches - home and away). Without loss of generality we assume that $T_{1}$ is "better than" $T_{2}$. This means that, in a set of matches, everything else equal, $T_{1}$ wins more matches against $T_{2}$. That is:

$$
\operatorname{Pr}\left(T_{1} \text { beats } T_{2}\right)>\operatorname{Pr}\left(T_{2} \text { beats } T_{1}\right) .
$$

If we restrict the analysis to decisive games, to avoid the draw option, we can simplify (2.1) to

$$
\operatorname{Pr}\left(T_{1} \text { beats } T_{2}\right)>\frac{1}{2}
$$

Let us define this probability (2.2) as $\alpha$. Let us furthermore assume that the two teams face a decision. Either they can keep on playing on the turf they have, which is natural grass, or they can change their pitch and substitute the natural to artificial turf. We name these two decisions $\mathcal{A}$ - changing to artificial and $\mathcal{N}$ staying with natural turf. 
Now, we make some strong simplifying assumptions. The playing strength ${ }^{2}$ of the team who chooses $\mathcal{A}$ increases relatively the opponent if the opponent chooses $\mathcal{N}$ and the match is played on artificial turf. We name this probabilistic advantage $\epsilon$. This also seems reasonable, as choosing $\mathcal{A}$ gives the team at hand the opportunity of training on this surface and, hence, improve their quality given this turf.

The final assumption is basically of a simplifying nature. It can, if necessary, be relaxed later. We assume that the team that chooses $\mathcal{N}$ does not gain a disadvantage when the match is played on natural turf, hopefully they keep the already acquired knowledge of natural turf play ${ }^{3}$.

Given the above assumptions, an underlying assumption of teams maximising the probability of a win, and straightforward probabilistic logic, the 2-player complete imperfect information game seen in Figure 1 can be formulated.

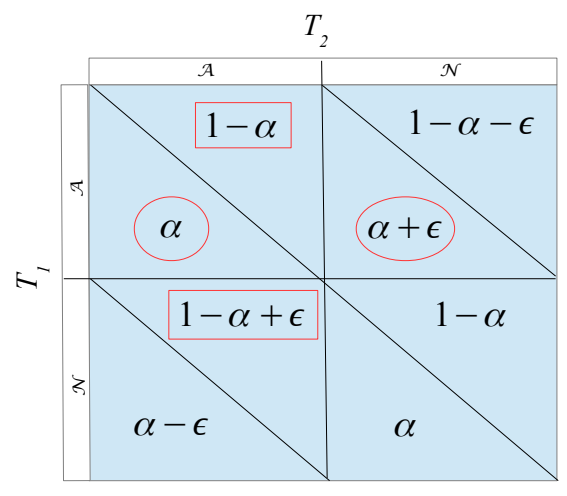

Figure 1. An initial and too simplified game formulation.

As Figure 1 indicates ${ }^{4}$, the Nash equilibrium is unique and straightforward, $\{\mathcal{A}, \mathcal{A}\}$, or both teams change to artificial turf. This is obviously a little too simple to catch up with some sense of reality. Some disadvantages by changing to turf must be present. Next, we discuss this model extension.

A change (choosing $\mathcal{A}$ ) involves some $\operatorname{costs}^{5}$. We assume furthermore that, for each team, such costs are different. This seems reasonable. A good team masters the art of playing football on natural turf better than a bad team. Let us name the cost consequence $\gamma_{i}, \forall i \in\{1,2\}$. As we see it, it also seems reasonable to

\footnotetext{
${ }^{2}$ Playing strength refers to the quality of one team versus another. So, a team with higher playing strength is simply assumed "better" than the opposing team.

${ }^{3}$ Obviously, the team playing on natural turf gains a disadvantage relative to the team playing on artificial turf due to probabilistic norming - each sub-square in Figure 1 must add up to 1. The point is that no extra disadvantage due to lower performance on natural grass by a artificial turf team is introduced. The only disadvantage, $-\epsilon$, introduced here is the advantage, $\epsilon$, gained by the artificial turf team due to probabilistic norming.

${ }^{4}$ Ellipses denote best reply for $T_{1}$ while rectangles denote best reply for $T_{2}$.

5 These costs are not necessarily related to the actual investment in the turf, but more related to the effect on playing strength.
} 
assume the following:

$$
\gamma_{1}>\gamma_{2}
$$

We can think of this assumption (2.3) in the following way: Playing good football is done by good football players with good coaches, and practice improves quality. Changing the turf should hence (relatively speaking) lead to a higher cost for the better team - both teams must in some sense start from scratch. That is, the comparative advantage gained by the better team is somewhat neutralized.

Now, in order to put this together we need to either convert costs to probabilities or probabilities for a win to revenues. It seems most convenient to do the first. Then, we do things as simple as possible, and just interpret the $\gamma$ 's as incremental probability decrease (or increase) instead of actual monetary costs. Given this assumption, we can formulate a slightly more realistic game version in Figure 2. Be aware of the probabilistic constraints that must hold - each sub-square must add up to 1 .

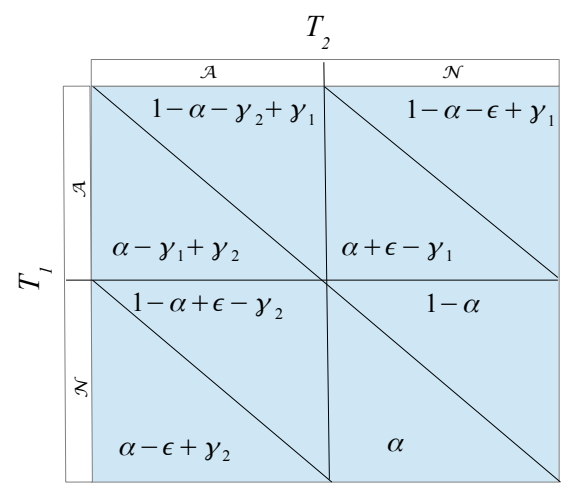

Figure 2. A more realistic game formulation.

Given the assumption (2.3), three possibilities exist ${ }^{6}$ :

(1) $\gamma_{1}>\epsilon>\gamma_{2}$,

(2) $\epsilon>\gamma_{1}>\gamma_{2}$,

(3) $\gamma_{1}>\gamma_{2}>\epsilon$.

Examining best replies ${ }^{7}$ for these three cases produces the output shown in Figure 2 .

As can be observed in Figure 2, Nash equilibria manifest themselves much in a fashion we would expect. Situation (3) is the 'status quo' situation where both good and bad teams stick to natural turf. Situation (2) might be defined as the long-run equilibrium outcome. Here, the benefit of artificial turf outweighs all costs, and as expected, both teams (or all teams) shift to artificial turf. One could think of this situation as a situation when artificial turf actually becomes as good as (or almost as good as) natural turf. Given reasonable assumptions on technological change, this is a situation we should expect. After all, if artificial

\footnotetext{
${ }^{6}$ Overlooking potential equalities for simplicity.

${ }^{7}$ Refer to appendix 4 for the math.
} 
(1)

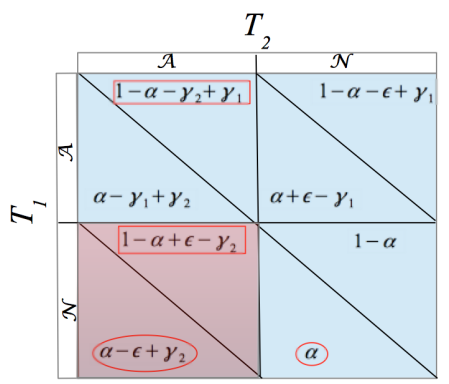

(2)

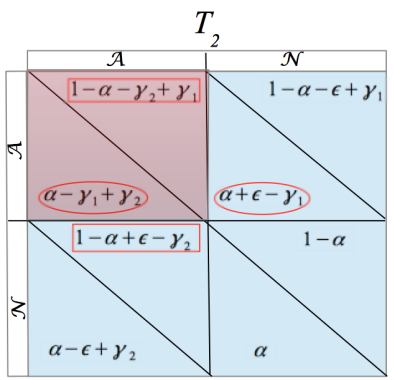

(3)

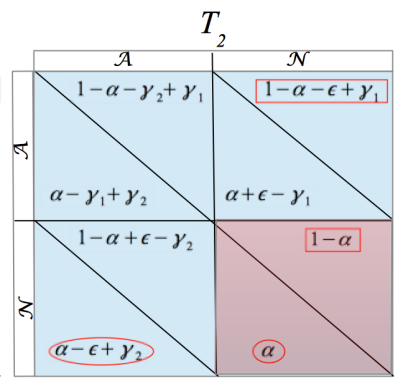

Figure 3. Best replies and Nash equilibria (red textured) for the cases (1), (2) and (3).

turf becomes comparable in play quality (as well as affordable) why would one continue to play on natural turf, given the obviously higher 'maintenance' costs.

The interesting situation is the one marked (1). Here, a certain trade-off between benefits and costs separate the two players (or if one likes groups of teams good and bad teams) into a pooling equilibrium. The good teams stick to natural turf, while the not so good teams choose to change to artificial turf. As discussed above, this seems most probably as a temporary solution, but it corresponds surprisingly well to some casual empiric's discussed below.

\section{Empirical analysis of Norway, Sweden and the Netherlands}

In order to test the "evolutionary status" of artificial turf in European football, a simple empirical analytic scheme is introduced. As the previous section indicated, in the transition period ${ }^{8}-$ situation (1) in Section 2, the model predicts that the best clubs (performance-wise) should stick to natural turf longer than the not so good clubs. Such a hypothesis can be tested empirically.

Let us assume that we define club performance by the so-called marathon table ${ }^{9}$. A marathon table contains aggregated point scores over a collection of several seasons. As such, teams' actual league performance over many seasons can be measured. Using marathon tables for Norway, Sweden and The Netherlands ${ }^{10},[3$, $4,6]$, as well as information on turf (natural/artificial) [1,2,12] in the top leagues for the 2017 and 2016/17 seasons, the following tables are constructed:

\footnotetext{
${ }^{8}$ Which clearly should be the present state as every team neither plays on natural nor artificial turf only.

${ }^{9}$ The choice of a marathon table as a proxy for separating good from bad teams may surely be questioned. A marathon table runs over long time periods, and a team that was "good" 20 years ago may definitely be "bad" today. On the other hand, the natural alternative of choosing shorter time horizons may also produce noise - proving too good quality for short lived successteams. Perhaps a financial proxy would have been better? However, the chosen leagues, those leagues where artificial turf has a reasonable spread involves serious measurement problems due to lack of accessible financial data. Hence, we landed on the marathon table as our chosen proxy.

${ }^{10}$ These countries are picked as they are the only European countries holding a significant share of matches played on artificial turf in the top league.
} 
Table 1. Data for Norwegian "Eliteserien", 2017 season. .

\begin{tabular}{lclc} 
Natural Turf & \multicolumn{3}{l}{ Artificial Turf } \\
\hline \hline Team & Rank & Team & Rank \\
\hline Brann & 4 & Molde FK & 6 \\
RBK & 1 & Vålerenga & 5 \\
LSK & 3 & Sarpsborg 08 & 23 \\
Haugesund & 22 & Strømsgodset & 11 \\
Stabæk & 13 & Troms $\varnothing$ & 10 \\
Sandefjord & 25 & Odd & 9 \\
Viking & 2 & Kristiansund & 44 \\
& & Aalesund & 21 \\
& & Sogndal & 20 \\
\hline \hline Average Rank & $\mathbf{1 0}$ & Average Rank & $\mathbf{1 6 . 6}$
\end{tabular}

Table 2. Data for Swedish "Allsvenskan", 2017 season. .

\begin{tabular}{lclc} 
Natural Turf & \multicolumn{3}{l}{ Artificial Turf } \\
\hline \hline Team & Rank & Team & Rank \\
\hline AIK & 3 & IFK Eskilstuna & 26 \\
Halmstads BK & 9 & BK Häcken & 18 \\
IFK Göteborg & 2 & Djurgårdens IF & 7 \\
IK Sirius & 34 & GIF Sundsvall & 24 \\
Jönköpings Södra & 27 & Hammarby IF & 12 \\
Kalmar FF & 14 & IF Elfsborg & 5 \\
Malmö FF & 1 & IFK Norrköping & 4 \\
& & Örebro SK & 11 \\
& & Östersunds FK & 35 \\
\hline \hline Average Rank & $\mathbf{1 2 . 9}$ & Average Rank & $\mathbf{1 5 . 8}$
\end{tabular}

Tables 1, 2 and 3 show that for the given seasons and countries, all "Natural turf teams" performed better - achieved lower average table rank - than "Artificial turf teams" 11 . Some simple statistical tests (one-tailed $t$-tests) do however reveal some lack of significance as the significance probabilities of the tests turned out to be $p=0.139, p=0.3148$ and $p=0.0357$ again for Norway, Sweden and Holland respectively. That is, with a normal assumption of $95 \%$ significance level, only the Dutch case proved significant. Still, our game model hypothesis is supported. Bad teams seem to choose artificial turf more quickly than good teams.

${ }^{11} 10<16.6,12.9<15.8$ and $8.9<16.2$ for Norway, Sweden and The Netherlands, respectively. 
Table 3. Data for Dutch "Eredivisie", 2016-17 season. .

\begin{tabular}{lclc} 
Natural Turf & \multicolumn{3}{l}{ Artificial Turf } \\
\hline \hline Team & Rank & Team & Rank \\
\hline Ajax & 1 & Heracles Almelo & 24 \\
Feyenoord & 3 & ADO Den Haag & 10 \\
PSV & 2 & Excelsior & 25 \\
Utrecht & 6 & PEC Zwolle & 23 \\
Vitesse & 13 & Sparta Rotterdam & 5 \\
AZ & 8 & Roda JC & 7 \\
FC Twente & 4 & & \\
FC Groningen & 11 & & \\
sc Herenveen & 16 & & \\
Wilhelm II & 12 & & \\
N.E.C. & 14 & & $\mathbf{1 6 . 2}$ \\
Go Ahead Eagles & 17 & &
\end{tabular}

\section{Discussion And Conclusions}

This paper has demonstrated that a relatively simple game model can explain the evolution of artificial turf in professional football. The identified Nash equilibria seem to fit the observed development of professional use of artificial turf pitches. In the initial phase - situation (3) in Figure 2 - no teams choose artificial turf, due to its quality and costs. Then, as quality increases and costs decrease, certain specific geographical locations ${ }^{12}$ end up in situation (1), where some teams choose artificial turf, others do not. As our model (and at least partially) our empirical examples indicate, the not so good teams. Then, finally, when artificial turf is both cheaper and in fact better (in all respects, play and injuries), situation (2) emerges, where all teams play on artificial turf. Clearly we are not there yet. However, given normal technology optimism, it is not much of a prediction to state that we will end up in situation (3), eventually.

Another, and interesting facet of situation (1), is a potential effect on uncertainty of outcome. As potined out by several authors, see for instance [10], this important sports economic parameter has shown alarming tendencies in major European football leagues the latter years. The obvious effect of some - not so good teams - starting to choose artificial turf, is a balancing effect in this dimension. As can be observed in Figure 2, the $\mathcal{A}$-strategy is a dominant strategy for Team 2. That is, Team 2, the not so good team, is better off by this choice than any other choice. Hence, it provides comparative advantage for Team 2 opposed to Team 1. Consequently, this force will work against decreased uncertainty of outcome.

\footnotetext{
${ }^{12}$ Winter in Norway and Sweden gives artificial turf and extra advantage.
} 


\section{Appendix A. Mathematical Derivation underlying Nash Equlibria IN FiguRE 2.}

Given (1) $\gamma_{1}>\epsilon>\gamma_{2}$, the 4 best reply correspondences are:

$$
\begin{array}{rlll}
\alpha-\epsilon+\gamma_{2}>\alpha-\gamma_{1}+\gamma_{2} & \Rightarrow \gamma_{1}>\epsilon & (\mathrm{OK}), \\
\alpha>\alpha+\epsilon-\gamma_{1} & \Rightarrow \gamma_{1}>\epsilon & (\mathrm{OK}), \\
1-\alpha-\gamma_{2}+\gamma_{1}>1-\alpha-\epsilon+\gamma_{1} & \Rightarrow \epsilon>\gamma_{2} & (\mathrm{OK}), \\
1-\alpha+\epsilon-\gamma_{2}>1-\alpha & \Rightarrow \epsilon>\gamma_{2} & (\mathrm{OK}) .
\end{array}
$$

Similarly, given $(2) \epsilon>\gamma_{1}>\gamma_{2}$ :

$$
\begin{array}{rlll}
\alpha-\epsilon+\gamma_{2}<\alpha-\gamma_{1}+\gamma_{2} & \Rightarrow \gamma_{1}<\epsilon & (\mathrm{OK}), \\
\alpha<\alpha+\epsilon-\gamma_{1} & \Rightarrow \gamma_{1}<\epsilon & (\mathrm{OK}), \\
1-\alpha-\gamma_{2}+\gamma_{1}>1-\alpha-\epsilon+\gamma_{1} & \Rightarrow \epsilon>\gamma_{2} & (\mathrm{OK}), \\
1-\alpha+\epsilon-\gamma_{2}>1-\alpha & \Rightarrow \epsilon>\gamma_{2} & (\mathrm{OK}) .
\end{array}
$$

Finally, given $(3) \gamma_{1}>\gamma_{2}>\epsilon$ :

$$
\begin{array}{rlll}
\alpha-\epsilon+\gamma_{2}>\alpha-\gamma_{1}+\gamma_{2} & \Rightarrow \gamma_{1}>\epsilon & (\mathrm{OK}), \\
\alpha>\alpha+\epsilon-\gamma_{1} & \Rightarrow \gamma_{1}>\epsilon & (\mathrm{OK}), \\
1-\alpha-\gamma_{2}+\gamma_{1}<1-\alpha-\epsilon+\gamma_{1} & \Rightarrow \epsilon<\gamma_{2} & (\mathrm{OK}), \\
1-\alpha+\epsilon-\gamma_{2}<1-\alpha & \Rightarrow \epsilon<\gamma_{2} & (\mathrm{OK}) .
\end{array}
$$

Hence, the Nash equilibria - as shown in Figure 2 - are correct.

\section{REFERENCES}

[1] Eliteserien i fotball for menn 2017 (in Norwegian), https://no.wikipedia.org/wiki/ Eliteserien_i_fotball_for_menn_2017\#Stadioner, 2017, accessed: 2018-08-13.

[2] Fotbollsallsvenskan 2017 (in Swedish), https://sv.wikipedia.org/wiki/Fotbollsall svenskan_2017, 2017, accessed: 2018-08-13.

[3] Allsvenska maratontabellen (in Swedish): 1924/25-2017, https://www2.svenskfotboll.se/ allsvenskan/historik/maratontabell/, 2018, accessed: 2018-08-13.

[4] Eredivisie, https://en.wikipedia.org/wiki/Eredivisie, 2018, accessed: 2018-08-13.

[5] Loftus Road, https://en.wikipedia.org/wiki/Loftus_Road, Aug 2018, accessed: 2018-0814.

[6] Maratontabellen (in Norwegian): 1949-2018, http://www.nifs.no/maraton.php, 2018, accessed: 2018-08-13.

[7] Stadiums with artifical turf, http://www.stadiumguide.com/figures-and-statistics/ lists/stadiums-with-artificial-turf/, Aug 2018, accessed: 2018-08-14.

[8] H. Andersson, B. Ekblom and P. Krustrup, Elite football on artificial turf versus natural grass: Movement patterns, technical standards, and player impressions, Journal of Sports Sciences 26 (2008), 113-122.

[9] P. Fleming and S. Forrester, Artificial turf research at Loughborough University, Procedia Engineering 72 (2014), 925-930.

[10] K. K. Haugen and K. P. Heen, The competitive evolution of European top football - signs of danger, European Journal of Sport Studies, 2017, doi: 10.12863/ejssax6x1-2018x1.

[11] L. M. Hvattum. Playing on artificial turf may be an advantage for Norwegian soccer teams, Journal of Quantitative Analysis in Sports 11 (2015), 183-192. 
[12] J. C. van Ours, Artificial pitches and unfair home advantage in professional football, Tinbergen Institute Discussion Paper TI 2017-093/V, Erasmus School of Economics, Erasmus University Rotterdam, Gustav Mahlerplein 117, 1082 MS Amsterdam, The Netherlands, 2017.

[13] S. Williams, P. A. Hume and S. Kara, A review of football injuries on third and fourth generation artificial turfs compared with natural turfs, Sports Medicine 41 (2011), 903-923.

Kjetil K. Haugen, Faculty of Business Administration and Social Sciences, Molde University College, Specialized University in Logistics, Britveien 2, 6410 Molde, Norway

e-mail: kjetil.haugen@himolde.no

Knut P. Heen, Faculty of Business Administration and Social Sciences, Molde University College, Specialized University in Logistics, Britveien 2, 6410 Molde, Norway

e-mail: knut.p.heen@himolde.no 
